\title{
Assessment Effect of Surface Modification of Nano Alumina with Vinyl Trimethoxy Silane to Mechanical Properties of Vinyl Ester Resin
}

\section{Rostam M*}

Department of Nanomaterials and Nanocoatings, Institute for Color Science and Technology, Tehran, P.O. Box 654-16764, Iran

\begin{abstract}
This paper reports Surface treatment of nano alumina with vinyl silane coupling agents was performed. Modification with silane coupling agents is commonly method by which the interfacial interaction of particles can be enhanced with resin. Surface treatment of nano alumina was implement under hydrolysis and condensation condition at different silane concentration. The surface characteristics of the unmodified and modified aluminum pigments were investigated by means of $\mathrm{CH}$ and FTIR analyses. The Mechanical properties of vinyl ester resin was investigated by the Dynamic mechanical thermal Analysis (DMTA). Results revealed that vinyl silane adsorbed on the surface of alumina under sol-gel reaction. Also, the mechanical properties of the vinyl ester composite was significantly enhanced with surface treated particles.
\end{abstract}

Keywords: Surface treatment; Silane coupling agent; Vinyl ester resin; DMTA

\section{Introduction}

In this study Surface treatment of nano alumina with vinyl silane coupling agents was performed. Modification with silane coupling agents is commonly method by which the interfacial interaction of particles can be enhanced with resins. Surface treatment of nano alumina was implement under hydrolysis and condensation condition at different silane concentration. Interactions of treated particles with resin taken place between the silane and Alumina, as well as the interactions between the organic parts of the silane with the polymeric matrix. Therefore, interfacial interaction of silane grafted Alumina plays a key role to ensure a better reinforcing effect. The present work is an attempt to study the interfacial bonding strength between differently vinyl silane treated nano Alumina and a vinyl ester matrix. This was based on the data deduced from dynamic mechanical thermal (DMTA) experiments of differently untreated and treated nano alumina loaded films. Results show that nano composite with 0.48 gr silane treated and $3 \% \mathrm{Wt}$. were in good agreement with the storage modulus and glass transition temperature values revealed by DMTA analysis.

\section{Experimental}

\section{Materials}

The spherical nano- $\mathrm{Al}_{2} \mathrm{O}_{3}$ particles, having average particle size Of $50 \mathrm{~nm}$, were provided from US Research Nanomaterials. VTMS with the chemical structure given in Figure 1 was purchased from Merck Co.

Epoxy Vinyl Ester Resin, Dimethyl Aniline (DMA), and Methyl Ethyl Ketone Peroxide (MEKP) as curing agents

Were obtained from Ashland Co.

\section{Surface modification of $\mathrm{Al}_{2} \mathrm{O}_{3}$ nanoparticles by VTMS}

The surface of $\mathrm{Al}_{2} \mathrm{O}_{3}$ nanoparticles was modified with VTMS. The surface modification was done using various amounts of APTMS. The lowest amount of silane used was calculated according to Eq. (1) [1].

$$
\mathrm{M}=\mathrm{M}_{\mathrm{p}} \times \mathrm{S}_{\mathrm{s}} / \mathrm{MSC}
$$

where $\mathrm{M}, \mathrm{Mp}$, Ss and MSC are the amount of silane (gr), the amount of nanoparticles (5 gr), the specific area of nanoparticles $\left(20 \mathrm{~m}^{2} / \mathrm{gr}\right)$ and the lowest surface coverage $\left(410 \mathrm{~m}^{2} / \mathrm{gr}\right)$, respectively. According to Eq. (1), it can be seen that the lowest amount of silane needed for surface modification of $5 \mathrm{gr}$ nanoparticles is $0.24 \mathrm{gr}$. According to Table 1, the specific amounts of the $\mathrm{Al}_{2} \mathrm{O}_{3}$ nanoparticles, ethanol, $\mathrm{H}_{2} \mathrm{O}$ and hydrochloric acid were placed in to a 3-neck reactor. The reactor was placed in an oil bath and the suspension was dispersed with a homogenizer for $2 \mathrm{~h}$. The suspension was kept under reflux at 70 $0 \mathrm{C}$ for $3 \mathrm{~h}$. The hydrolysis ratio for hydrolysis reaction is 27 .

In this study the nanoparticles were treated by $0.24,0.48$ and $1 \mathrm{gr}$ Vinyl trimethoxy silane. The $\mathrm{pH}$ of hydrolysis was adjusted at around 3-4 by Acetic acid and the react ion was continue for $2 \mathrm{~h}$. After the hydrolysis, sodium hydroxide (50\%) was then added to the suspension and the $\mathrm{pH}$ was adjusted around 8-9 and the condensation react ion long for $3 \mathrm{~h}$. Finally, the slurries were separated from solvent by centrifuge. The residue was washed with water and ethanol for six times in order to ensure that all unreacted silanes are carefully removed. The precipitates were dispersed in the mixture of ethanol and $\mathrm{H}_{2} \mathrm{O}$ followed by drying with a spray dryer.

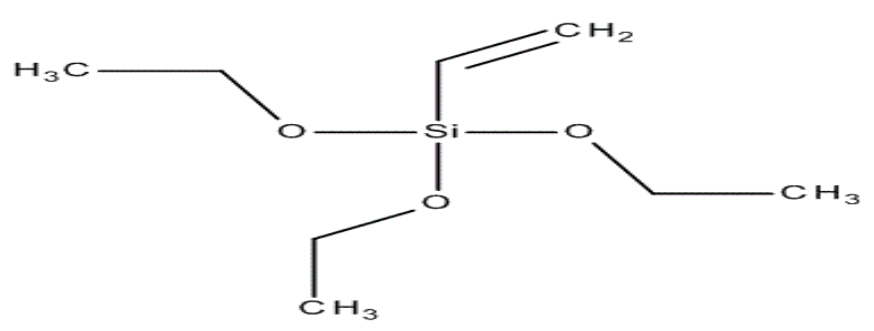

Figure 1: Structural formulae vinyl tri methoxy silane (VTMS)

*Corresponding author: Rostam M, Department of Nanomaterials and Nanocoatings, Institute for Color Science and Technology, Tehran, P.O. Box 65416764, Iran, Tel: 98212296 9771; E-mail: me@ehsan.it

Received April 06, 2016; Accepted April 13, 2016; Published April 20, 2016

Citation: Rostam M (2016) Assessment Effect of Surface Modification of Nano Alumina with Vinyl Trimethoxy Silane to Mechanical Properties of Vinyl Ester Resin. J Nanomed Nanotechnol 7: 365. doi:10.4172/2157-7439.1000365

Copyright: (c) 2016 Rostam M. This is an open-access article distributed under the terms of the Creative Commons Attribution License, which permits unrestricted use, distribution, and reproduction in any medium, provided the original author and source are credited. 


\section{Preparation of vinyl ester nanocomposite coatings}

Weight loading $3 \mathrm{wt} . \%$ of modified and unmodified nanoparticles were dispersed in Vinyl Ester resin using zirconia pearl. Then curing agent, Cobalt, DMA and MEKP with 0.7, 0.2 and 1.2 wt.\% were added to the Vinyl ester/nanoparticles mixture. The mixtures intensively mixed by mixer for $3 \mathrm{~min}$. The nanocomposites prepared were applied on the cleaned siliconized sheets using film applicator. Finally, coatings were cured at $23 \pm 2^{\circ} \mathrm{C}$ for $24 \mathrm{~h}$. The dry thickness of the coating was $1000 \pm 50 \mu \mathrm{m}$.

\section{Results and Discussion}

The Nano aluminia powder was modified with Vinyl-tri-(methoxy silane). Figure 1 shows the general chemical structure of the Vinyl Trimethoxy silane.

The adsorption of Vinyl Trimethoxy silane on the surface of Nano alumina powder was studied by FTIR analysis (Figure 2) and $\mathrm{CH}$ analysis. Results show two adsorption bands in the wavenumber regions ranging from $1350 \mathrm{~cm}^{-1}$ to $1450 \mathrm{~cm}^{-1}$ corresponding to the presence of $\mathrm{C}=\mathrm{C}$ groups on the aluminium powder surface. C-H stretching vibrations appeared at $2900-2950 \mathrm{~cm}^{-1}$. All of these observations confirm the adsorption of Vinyl trimethoxy layer on the alumina surface.

To estimating the amount of VTMS grafted on the surface of alumina $\mathrm{CH}$ analysis was done. The test was carried out on the unmodified and modified particles. Results of $\mathrm{CH}$ analysis are given in Table 2. According to Table 2, weight percent 0.47-1.41 for carbon and 0.17-0.36 for hydrogen was observed for the modified alumina particles compared to the unmodified.

This confirms the results of FTIR that VTMS could successfully adsorb on the alumina surface. Also, according to these results deduced the increasing of silane concentration caused further silane grafting on alumina surface.

To investigating interfacial interaction of nano alumina with vinyl ester resin, DMTA analysis carried out. These tests were implemented on the coatings containing nano particles with different grafting content. Plots of tan delta versus temperature and storage modulus versus temperature of the samples are shown in Figures 3 and 4.

Results showed that the mechanical properties of the Vinyl Ester coating were significantly improved after adding modified

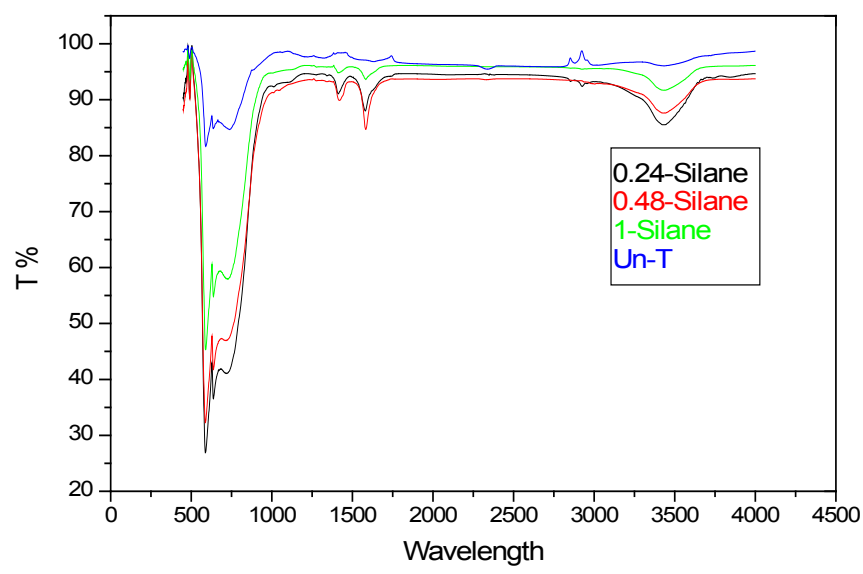

Figure 2: FTIR spectra of vinyl silane layers on nano alumina.

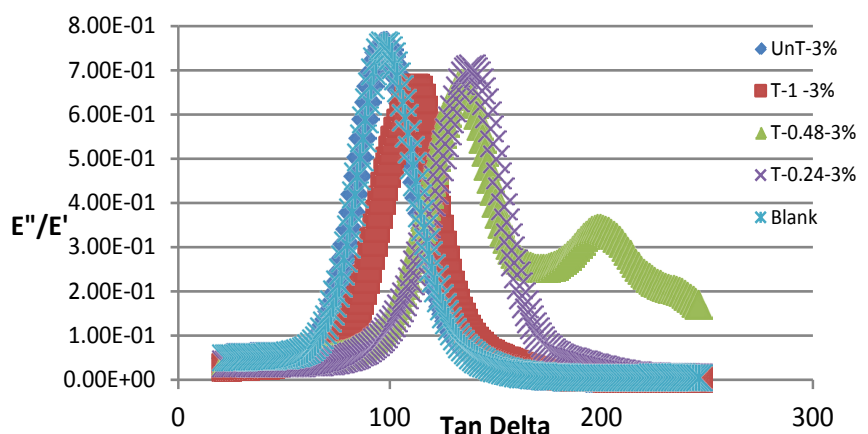

Figure 3: Variations of Tan delta versus temperature of the coatings loaded with modified and unmodified Particles.

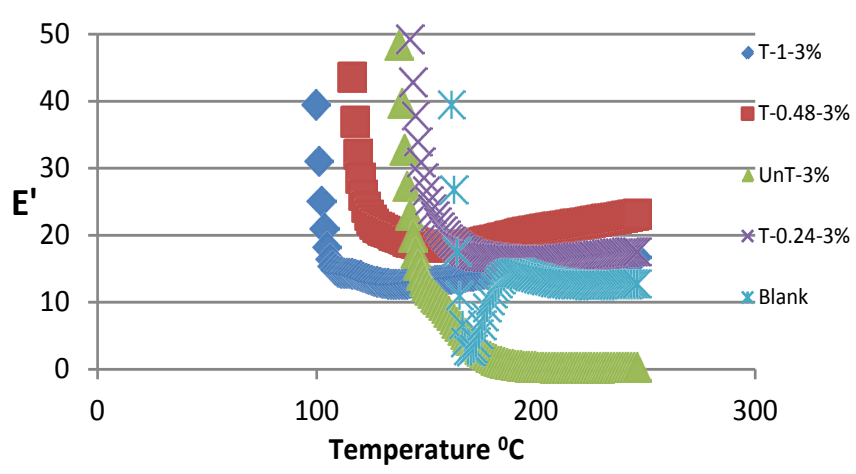

Figure 4: Variations of storage modulus versus temperature of the coatings loaded with modified and unmodified particles.

\begin{tabular}{|c|c|c|c|c|c|}
\hline $\begin{array}{c}\mathbf{p H} \text { in } \\
\text { condensation } \\
\mathbf{s t e p}\end{array}$ & $\begin{array}{c}\mathbf{N a O H} \\
\mathbf{( 5 0} \mathbf{~ \% )}\end{array}$ & $\begin{array}{c}\mathbf{p H} \text { in } \\
\text { hydrolysis step }\end{array}$ & $\begin{array}{c}\text { Acetic } \\
\text { Acid } \\
\mathbf{( 3 7 \% )}\end{array}$ & $\mathbf{H}_{\mathbf{2}} \mathbf{O}$ (gr) & Silane (gr) \\
\hline 10-Aug & $0.2 \mathrm{cc}$ & 4-Mar & $0.6 \mathrm{cc}$ & 0.58 & 0.24 \\
\hline 10-Aug & $0.4 \mathrm{cc}$ & $4-M a r$ & $1.4 \mathrm{cc}$ & 1.215 & 0.48 \\
\hline 4-Mar & 0.8 & 4-Mar & $2.8 \mathrm{cc}$ & 2.55 & 1 \\
\hline
\end{tabular}

Table 1: Amount of materials used.

\begin{tabular}{|c|c|c|}
\hline Sample & $\mathbf{C} \%$ & $\mathbf{H} \%$ \\
\hline Al-0.24 & 0.47 & 0.17 \\
\hline Al-0.48 & 1.39 & 0.33 \\
\hline Al-1.0 & 1.41 & 0.36 \\
\hline
\end{tabular}

Table 2: $\mathrm{CH}$ analysis for modified nano alumina.

nanoparticles. This show that surface grafting of silane on the surface of particles could result in better physical/chemical interactions with the resin matrix.

For the nano composite containing $3 \mathrm{w}$ t.\% of nanoparticles modified with $0.48 \mathrm{gr}$ silane, improvement in the mechanical properties was greater than those nanoparticles modified with $0.24,1 \mathrm{gr}$ silane.

These observations reveal that nanoparticles influenced curing behavior of the polyurethane coating [2]. This may be attributed to the steric hindrance effect of nanoparticles during curing reaction or their effect on increasing coating viscosity $[3,4]$.

These are responsible for difficulties for the coating functional groups reaction. The reaction of the reactive groups of the binder with the vinyl group of the silane may also result in cross-linking density increment. Results also show that coatings reinforced with $0.48 \mathrm{gr}$ 
silane modified nanoparticles showed greater cross-linking densities than the other loaded modified particles. This again reveals that surface modification of the particles affected coating viscoelastic properties. In fact, the modified particles could form stronger bonds with the coating matrix causing a lower phase separation and higher compatibility. The higher cross-linking density and $\mathrm{Tg}$ of the coating loaded with $3 \mathrm{wt} \%$ nanoparticles, modified with 0.48 gr silane, than modified with $0.24,1$ gr silane samples is the main reason for better mechanical strength of this sample than others.

\section{Conclusion}

Alumina powder was modified with the Vinyl trimethoxy silane layer. The results obtained from FTIR and CH

Analyses revealed that the Vinyl tri methoxy silane successfully adsorbed on the surface of nano alumina via chemical interaction.

Generally, the result obtained from DMTA analysis confirmed that addition of nanoparticles caused a decrease in the coating chemical cross-linking density due to the steric hindrance effects of the nanoparticles on the curing behavior of the coating [5]. Moreover, the decrease in cross-linking density was less pronounced when the surface modified nanoparticles were added to the coating. In fact, the pure polyurethane coating (with nanoparticles) shows more brittleness than the one with surface modified nanoparticles. Nanoparticles could reduce chemical cross-links but increase physical/chemical bonds with the coating matrix $[5,6]$. As a result, nano particles behave like toughening agents causing better coating capability of resisting against stress.

\section{References}

1. Palimi MJ, Rostami M, Mahdavian M, Ramezanzadeh B (2014) Surface modification of $\mathrm{Fe}_{2} \mathrm{O}_{3}$ nanoparticles with 3-aminopropyltrimethoxysilane (APTMS): An attempt to investigate surface treatment on surface chemistry and mechanical properties of polyurethane/ $\mathrm{Fe}_{2} \mathrm{O}_{3}$ nanocomposites. Applied Surface Science 320: 60-72.

2. Kruenate J, Aumnate C, Tangjuntra N, Sooksomsong T, Kongniam S (2011) The rheological properties of interface/morphology relationships of Hemp fiberreinforced thermoplastic starch composites. Coffee Break and Poster Session 2-10: 40-12: 10

3. Sinko J (2001) Challenges of chromate inhibitor pigments replacement in organic coatings. Prog Org Coat 42: 267-282.

4. Sabzi M, Mirabedini SM, Atai M (2009) Surface modification of $\mathrm{TiO}_{2}$ nanoparticles with silane coupling agent and investigation of its effect on the properties of polyurethane composite coating. Prog Org Coat 65: 222-228.

5. Li JH, Hong RY, Li MY, Li HZ, Zheng Y, et al. (2009) Effects of ZnO nanoparticles on the mechanical and antibacterial properties of polyurethane coatings Prog Org Coat 64: 504-509.

6. Rostami M, Mohseni M, Ranjbar Z (2012) An attempt to quantitatively predict the interfacial adhesion of differently surface treated nanosilicas in a polyurethane coating matrix using tensile strength and DMTA analysis. Int $\mathrm{J}$ Adhes Adhes 34: 24-31. 